\title{
Rotators for matching non-symmetric ion-therapy beams to rotating gantries
}

\author{
Márius Pavlovič* and Jozef Bokor \\ Institute of Nuclear and Physical Engineering, Faculty of Electrical Engineering and Information Technology, \\ Slovak University of Technology in Bratislava \\ Ilkovičova 3, 81219 Bratislava, Slovak Republic \\ *marius.pavlovic@stuba.sk \\ †jozef.bokor@stuba.sk \\ Andrea Šagátová \\ University Centre of Electron Accelerator, Slovak Medical University, Ku Kyselke 497 \\ 91106 Trenčin, Slovak Republic \\ andrea.sagatova@stuba.sk \\ Published 1 September 2016
}

\begin{abstract}
The paper deals with special ion-optical matching sections called "rotators" for matching nonsymmetric beams to rotating ion-therapy gantries. General matrix analysis of the problem is formulated resulting in a specific set of ion-optical constraints that must be fulfilled by the rotator transfer matrix. Possible ways of fitting these ion-optical constraints are discussed and illustrated by several examples of suitable rotator lattices. Each lattice is representing a different type of rotator, e.g. point-to-point imaging lattice or parallel-to-point imaging lattice. Optimization of the rotator lattice with respect to its total length is discussed, and the most compact solutions are presented as well.
\end{abstract}

Keywords: Gantry; ion therapy; rotator.

PACS numbers: 87.56.bd, 29.27.-a, 41.85.-p.

\section{Introduction}

Modern proton and ion therapy facilities have an ambition to use the most advanced beam delivery techniques in order to achieve the best dose distribution in the patient body. These beam delivery techniques use rotating gantries equipped with pencil-beam scanning. ${ }^{1}$ The gantry is a terminating part of the beam line transporting the beam from the accelerator to the patient. It is mechanically rotated around the incoming beam axis in order to direct the beam to the patient from any angle as specified by a physician in the treatment plan. Combining a rotating gantry with pencil-beam scanning becomes an ionoptical challenge, especially in the case when an accelerator is serving a non-symmetric beam. A "non-symmetric beam" stands for a beam with different emittance patterns in

This is an Open Access article published by World Scientific Publishing Company. It is distributed under the terms of the Creative Commons Attribution 3.0 (CC-BY) License. Further distribution of this work is permitted, provided the original work is properly cited. 
the two transverse planes. $^{2}$ There are several possibilities of matching these nonsymmetric beams to rotating gantries. ${ }^{3-5}$ In this paper, the emphasis is paid to a technique based on a special matching section called the rotator. It has been reinvented by the PIMMS group ${ }^{6}$ at CERN (Proton Ion Medical Machine Study) following its sketched version in the US Patent on a Multi-Station Proton Beam Therapy System. ${ }^{7}$ Rotator matching is the most universal matching technique suitable for any gantry type and any incoming beam. However, it requires an additional dedicated section in the high energy beam transfer line that must be mechanically rotated as a function of the gantry rotation.

So far, there is only a single installation of a rotator worldwide, namely at the MedAustron facility in Austria. ${ }^{8}$ This rotator possesses a special unit-like transfer matrix in order to meet the modular philosophy of the beam transport design. ${ }^{9}$ We expanded our study to a broader spectrum of possible rotator lattices satisfying a generally formulated set of ion-optical constraints. This made it possible to shorten the rotator length, which may be an important point especially for compact hospital-based ion-therapy facilities.

\section{Ion-optical Formulation of the Problem using the Matrix Formalism}

The principle of the rotator matching can be explained using the standard matrix formalism of beam transport. ${ }^{10,11}$ A rotator is a bending-free, dispersion-free, and coupling-free section of the transfer line featuring a special transfer matrix, $\mathbf{M}_{\mathbf{R O T}}$, in the form (it is sufficient to consider a $4 \times 4$ matrix for the two transverse planes):

$$
\mathbf{M}_{\mathbf{R O T}}=\left(\begin{array}{rlrl}
r_{11} & r_{12} & 0 & 0 \\
r_{21} & r_{22} & 0 & 0 \\
0 & 0 & -r_{11} & -r_{12} \\
0 & 0 & -r_{21} & -r_{22}
\end{array}\right) .
$$

All terms in the vertical sub-matrix must be a negative "mirror" of the corresponding terms in the horizontal sub-matrix. Now, let us assume that the rotator is rotated by an angle $\alpha$ with respect to the exit of the fixed beam line, and the gantry is rotated by another angle $\alpha$ with respect to the exit of the rotator. This practically means that the gantry is rotated by the angle $2 \alpha$ with respect to the exit of the fixed beam line. The situation is illustrated in Fig. 1.

The overall transfer matrix, $\mathbf{M}$, from the exit of the fixed beam line to the entrance of the gantry will be:

$$
\mathbf{M}=\mathbf{M}_{\boldsymbol{\alpha}} \mathbf{M}_{\mathbf{R O T}} \mathbf{M}_{\boldsymbol{\alpha}},
$$

where $\mathbf{M}_{\boldsymbol{\alpha}}$ is the coordinate system rotation matrix:

$$
\mathbf{M}_{\alpha}=\left(\begin{array}{cccc}
\cos \alpha & 0 & \sin \alpha & 0 \\
0 & \cos \alpha & 0 & \sin \alpha \\
-\sin \alpha & 0 & \cos \alpha & 0 \\
0 & -\sin \alpha & 0 & \cos \alpha
\end{array}\right) .
$$




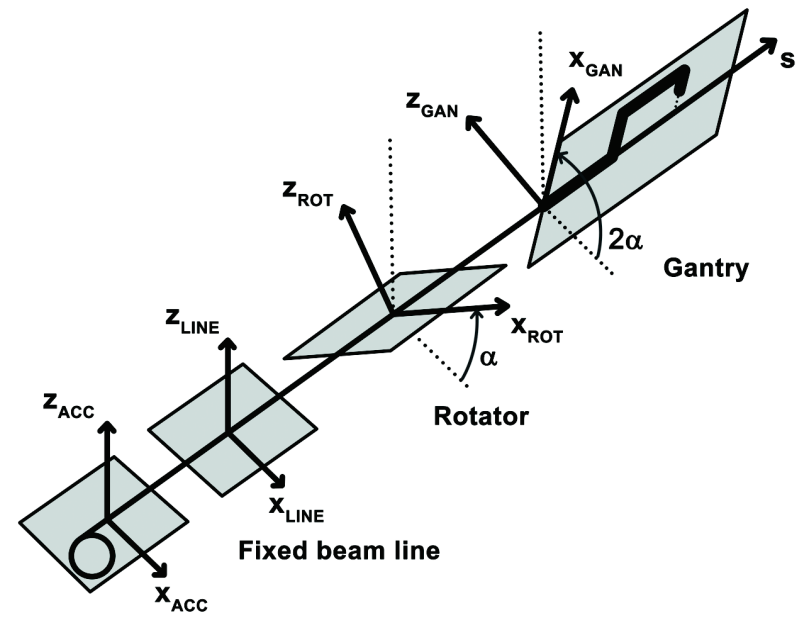

Accelerator with

extraction line

Fig. 1. Schematic illustration of a beam line with a rotator and a gantry.

It can be shown by performing the matrix multiplication in Eq. (2) that the overall transfer matrix M becomes identical to the rotator matrix Мвот. Let us demonstrate this for the " 11 " and " 13 " terms of the overall transfer matrix, $m_{11}$ and $m_{13}$, respectively:

$$
\begin{gathered}
m_{11}=r_{11} \cos ^{2} \alpha+r_{11} \sin ^{2} \alpha=r_{11}, \\
m_{13}=r_{11} \cos \alpha \sin \alpha-r_{11} \cos \alpha \sin \alpha=0 .
\end{gathered}
$$

The other terms behave in the same way. This practically means that the beam parameters at the entrance of the gantry are not a function of the gantry angle, which is the main role of the rotator. The rotator maps the beam parameters from the exit of the fixed beam line to the entrance of the gantry (and possibly transforms them according to the rotator transfer matrix). In this configuration, the gantry "sees" always the same input beam parameters independently from the rotation angle. It is, however, a challenge to find suitable lattices satisfying Eq. (1). We have developed an effective strategy of fitting the terms of the rotator transfer matrix. This strategy is going to be presented and demonstrated in the next sections.

\subsection{Fitting the rotator transfer-matrix}

According to our experience, an effective strategy for fitting the terms of the rotator transfer matrix can be based on the Twiss formalism. ${ }^{11}$ If there is no coupling between the two transverse planes, both (horizontal and vertical) transfer sub-matrices can be written in terms of the Twiss parameters as: 


$$
\mathbf{M}_{\mathbf{R O T}}^{h, v}=\left(\begin{array}{cc}
\sqrt{\frac{\beta_{2}^{h, v}}{\beta_{1}^{h, v}} \cos \mu^{h, v}+\alpha_{1}^{h, v} \sin \mu^{h, v}} & \sqrt{\beta_{1}^{h, v} \beta_{2}^{h, v}} \sin \mu^{h, v} \\
\frac{\left(\alpha_{1}^{h, v}-\alpha_{2}^{h, v}\right) \cos \mu^{h, v}-\left(1+\alpha_{1}^{h, v} \alpha_{2}^{h, v}\right) \sin \mu^{h, v}}{\sqrt{\beta_{1}^{h, v} \beta_{2}^{h, v}}} & \sqrt{\frac{\beta_{1}^{h, v}}{\beta_{2}^{h, v}} \cos \mu^{h, v}-\alpha_{2}^{h, v} \sin \mu^{h, v}}
\end{array}\right)
$$

where $\beta$ is the beta-function, $\mu$ is the phase advance, indices " 1 " and " 2 " refer to the entrance and the exit of the rotator, respectively, and indices " $h$ " and " $v$ " refer to the horizontal and vertical plane, respectively. The following relations hold:

$$
\mu=\int_{1}^{2} \frac{1}{\beta(s)} d s \quad \text { and } \quad \alpha(s)=-\frac{1}{2} \frac{d \beta(s)}{d s},
$$

where $s$ is the longitudinal coordinate along the design orbit.

In order to simplify the fitting strategy, we set $\alpha_{1}=0$ in both transverse planes and asked the fitting routine to match $\alpha_{2}=0$, too (waist-to-waist). With this simplification, the following relations must hold for the rotator transfer matrix:

$$
\begin{gathered}
\sqrt{\frac{\beta_{2}^{h}}{\beta_{1}^{h}}} \cos \mu^{h}=-\sqrt{\frac{\beta_{2}^{v}}{\beta_{1}^{v}}} \cos \mu^{v}, \\
\sqrt{\beta_{1}^{h} \beta_{2}^{h}} \sin \mu^{h}=-\sqrt{\beta_{1}^{v} \beta_{2}^{v}} \sin \mu^{v}, \\
-\frac{\sin \mu^{h}}{\sqrt{\beta_{1}^{h} \beta_{2}^{h}}}=\frac{\sin \mu^{v}}{\sqrt{\beta_{1}^{v} \beta_{2}^{v}}}, \\
\sqrt{\frac{\beta_{1}^{h}}{\beta_{2}^{h}}} \cos \mu^{h}=-\sqrt{\frac{\beta_{1}^{v}}{\beta_{2}^{v}}} \cos \mu^{v} .
\end{gathered}
$$

\section{Results}

The terms of the transfer matrix can be fitted in a systematic way. Different fitting strategies correspond to different types of rotators. The first type is a point-to-point imaging lattice.

\subsection{Point-to-point imaging lattice}

The point-to-point imaging lattice is generally characterized by $r_{12}=r_{34}=0$. The relations in Eq. (8a-8d) translate into the following set of constraints:

$$
\begin{gathered}
\sqrt{\frac{\beta_{2}^{h}}{\beta_{1}^{h}}} \cos \mu^{h}=-\sqrt{\frac{\beta_{2}^{v}}{\beta_{1}^{v}}} \cos \mu^{v}, \text { and } \\
\sin \mu^{h}=\sin \mu^{v}=0,
\end{gathered}
$$

which can be satisfied if the phase advance is an integer multiple of $\pi$ and the horizontal and vertical phase advances are shifted by $\pi$ with respect to each other. The beta functions must satisfy the condition: 


$$
\frac{\beta_{2}^{h}}{\beta_{1}^{h}}=\frac{\beta_{2}^{v}}{\beta_{1}^{v}} .
$$

We designed two structures of this type. In the first structure, the output-to-input ratio of beta functions is 1 , the horizontal phase advance is $2 \pi$, and the vertical phase advance is $\pi$. Such a rotator maps the beam 1:1 from the exit of the fixed beam line to the entrance of the gantry. Figure 2 shows the corresponding beta functions.

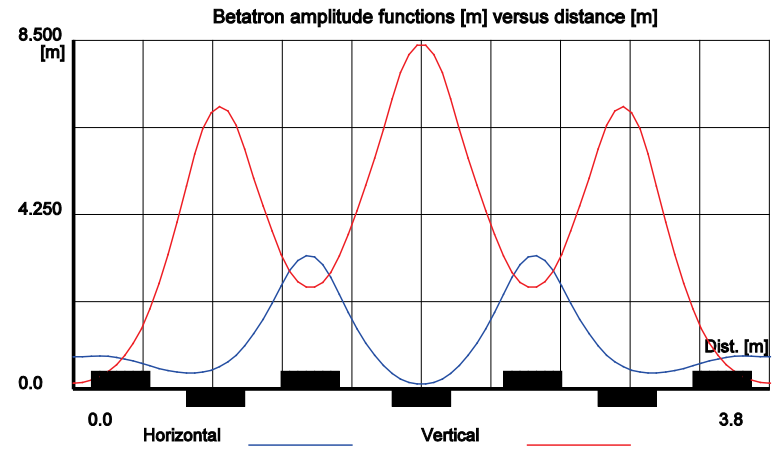

Fig. 2. Beta functions of the first version of the rotator with the unit transfer matrix. Quadrupoles are indicated by the black boxes: upper orientation - a horizontally focusing quadrupole; bottom orientation - a vertically focusing quadrupole.

The seven quadrupoles of this structure are grouped into four families Q1=Q7, $\mathrm{Q} 2=\mathrm{Q} 6, \mathrm{Q} 3=\mathrm{Q} 5$ and $\mathrm{Q} 4$. This reflects the symmetry of the Twiss parameters at the entrance and exit of the rotator.

The second structure was matched for the output-to-input ratio of beta functions different from one, namely 0.5 . The number of quadrupoles has been reduced to six, but the quadrupole families were abandoned. The structure got slightly shorter than the previous one. The resulting beta functions are shown in Fig. 3.

\subsection{Parallel-to-point imaging lattice}

The parallel-to-point imaging is characterized by $r_{11}=\mathrm{r}_{33}=0$. The relations in Eq. (8a-8d) translate into the following set of constraints:

$$
\begin{gathered}
\cos \mu^{h}=\cos \mu^{v}=0, \text { and } \\
\sqrt{\beta_{1}^{h} \beta_{2}^{h}} \sin \mu^{h}=-\sqrt{\beta_{1}^{v} \beta_{2}^{v}} \sin \mu^{v},
\end{gathered}
$$

which can be satisfied if the phase advance is an integer multiple of $\pi / 2$ and the horizontal and vertical phase advances are again shifted by $\pi$ with respect to each other. The beta functions must satisfy the condition:

$$
\beta_{1}^{h} \beta_{2}^{h}=\beta_{1}^{v} \beta_{2}^{v} .
$$




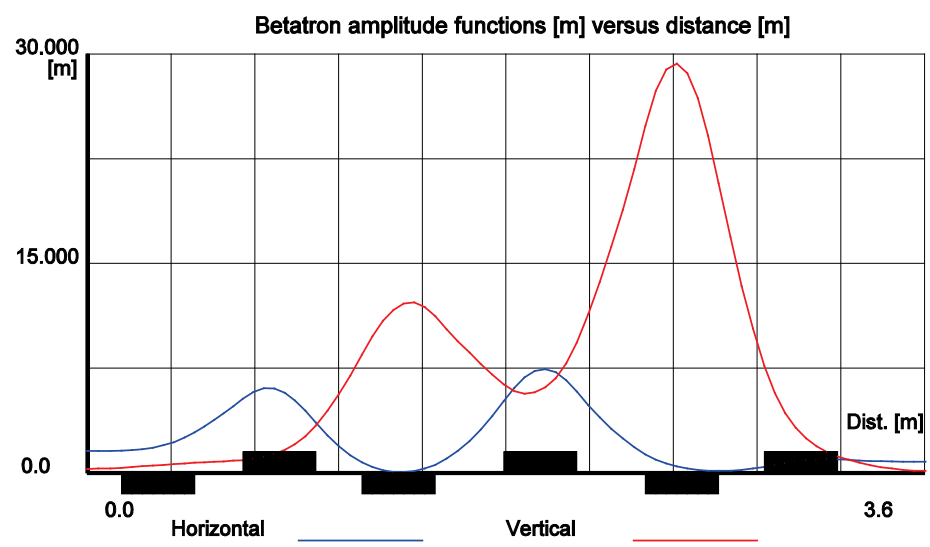

Fig. 3. Beta functions of the second version of the rotator. Quadrupoles are indicated by the black boxes: upper orientation - a horizontally focusing quadrupole; bottom orientation - a vertically focusing quadrupole.

Because of the smaller phase advance, a structure shorter than the point-to-point imaging lattice should be expected. As an example, a lattice with the horizontal phase advance $3 \pi / 2$ and the vertical phase advance $\pi / 2$ is presented. It consists of five quadrupoles grouped into three families $\mathrm{Q} 1=\mathrm{Q} 5, \mathrm{Q} 2=\mathrm{Q} 4$ and $\mathrm{Q} 3$. The corresponding beta functions are plotted in Fig. 4. This rotator is $\approx 1 \mathrm{~m}$ shorter than the point-to-point one.

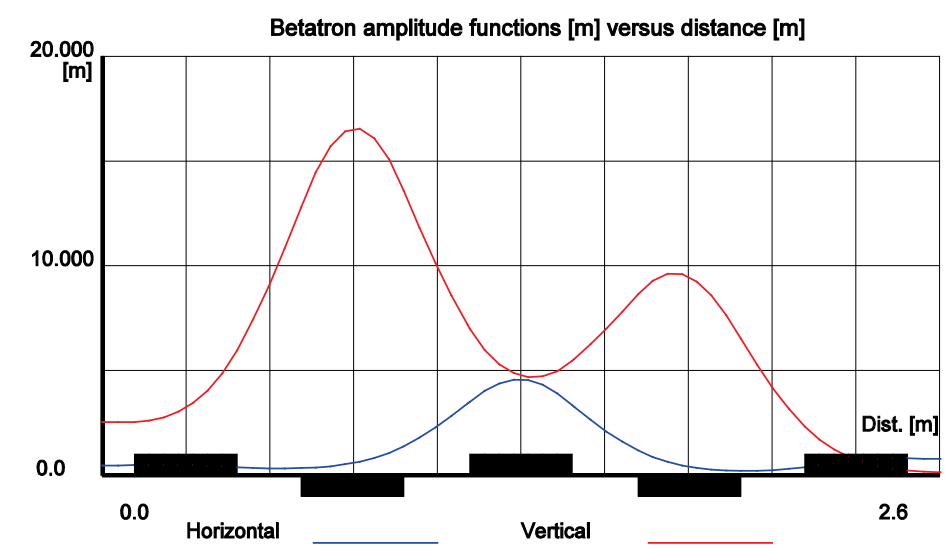

Fig. 4. Beta functions of the parallel-to-point rotator. Quadrupoles are indicated by the black boxes: upper orientation - a horizontally focusing quadrupole; bottom orientation - a vertically focusing quadrupole.

\subsection{General rotator lattice}

In a general case, the relations in Eq. (8a-8d) translate into the following set of constraints: 


$$
\begin{gathered}
\sqrt{\frac{\beta_{2}^{h}}{\beta_{1}^{h}}} \cos \mu^{h}=-\sqrt{\frac{\beta_{2}^{v}}{\beta_{1}^{v}}} \cos \mu^{v}, \text { and } \\
\sqrt{\beta_{1}^{h} \beta_{2}^{h}} \sin \mu^{h}=-\sqrt{\beta_{1}^{v} \beta_{2}^{v}} \sin \mu^{v},
\end{gathered}
$$

which can be satisfied if the horizontal and vertical phase advances are shifted by $\pi$ with respect to each other and the beta functions simultaneously satisfy the conditions:

$$
\begin{aligned}
\sqrt{\frac{\beta_{2}^{h}}{\beta_{1}^{h}}} & =\sqrt{\frac{\beta_{2}^{v}}{\beta_{1}^{v}}}, \\
\sqrt{\beta_{1}^{h} \beta_{2}^{h}} & =\sqrt{\beta_{1}^{v} \beta_{2}^{v}} .
\end{aligned}
$$

Solving the set in Eq. (14a-14b) yields:

$$
\beta_{1}^{h}=\beta_{1}^{v} \cap \beta_{2}^{h}=\beta_{2}^{v},
$$

which means that one has to set identical input beta functions in the two transverse planes and match identical output beta functions in the two transverse planes. The input and output beta functions need not to be identical. Nevertheless, we selected an example of a special case with the same input and output beta functions (see Fig. 5).

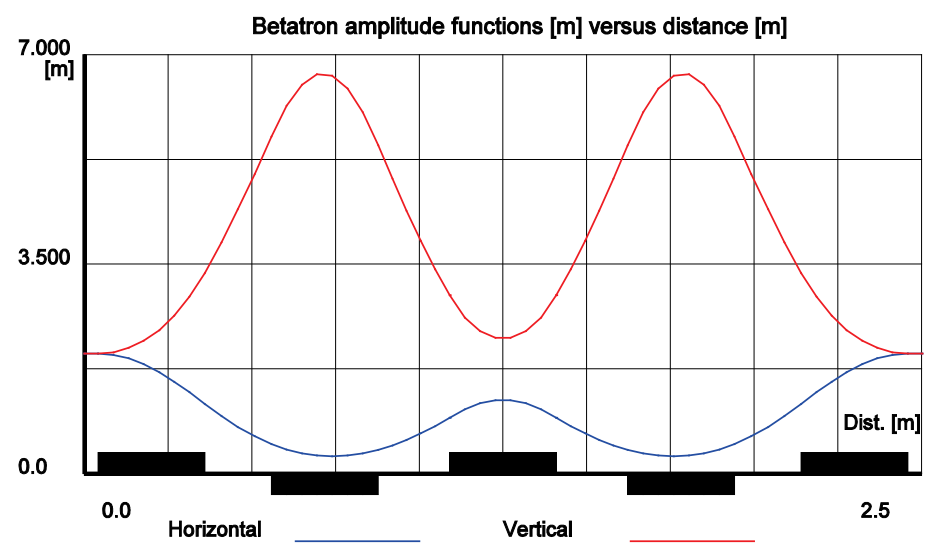

Fig. 5. Rotator with identical beta functions. Quadrupoles are indicated by the black boxes: upper orientation a horizontally focusing quadrupole; bottom orientation - a vertically focusing quadrupole.

The rotator consists of 5 quadrupoles grouped into three families Q1=Q5, Q2=Q3, Q4. The horizontal phase advance is 3.883852 , the vertical phase advance is 0.742259 , and the structure is $2.514 \mathrm{~m}$ long.

\section{Discussion and Conclusions}

All rotators presented in this paper have been designed by the WinAGILE beam transport code written by Phil Bryant. Fitting the ion-optical constraints was done by its built-in matching routine, and we observed an excellent converging capability in most cases. In 
all designs, the feasibility of all beam-transport elements has been checked. The quadrupoles have an effective length of $0.32 \mathrm{~m}$ and a maximum field on the pole-tip of $1 \mathrm{~T}$. The quadrupole half-aperture is $4 \mathrm{~cm}$, which corresponds to the maximum magnetic field gradient of $25 \mathrm{~T} / \mathrm{m}$. Each rotator has also been checked for the beam envelopes to make sure that they do not exceed the quadrupoles` aperture.

There are two essential features of our fitting strategy: (a) the fitting is done assuming the waist-to-waist imaging, and (b) the horizontal and vertical phase advances are shifted by $\pi$ with respect to each other. This provides a good compromise between the complexity and flexibility of the ion-optical design. The waist-to-waist condition makes the terms of the transfer matrix sin-like or cos-like functions of the phase advance. This can be used for the necessary sign-inversion between the horizontal and vertical submatrices of the rotator transfer matrix simply by shifting the horizontal and vertical phase advances by $\pi$ with respect to each other. The absolute values of the cos-like terms as well as the sin-like terms are the same. This simplifies the associated constraints for the beta functions. Despite this simplicity, different types of rotators could be designed including point-to-point imaging and parallel-to-point imaging ones. These special cases are suitable for the modular philosophy of the beam-transport system. ${ }^{9}$

As expected, the structures with smaller phase advance are, in general, shorter than the structures with larger phase advance. Compactness of the rotator may be important for space-saving reasons as well as for minimizing misalignments in a mechanically rotated structure.

\section{Acknowledgments}

This work was supported by the Slovak Science and Research Agency under the contract number APVV-0321-11. We would like to express our compliment to Phil Bryant for writing an excellent and enjoyable WinAGILE beam transport code.

\section{References}

1. T. Haberer, W. Becher, D. Schardt and G. Kraft, Nucl. Instr. Meth. Phys. Res. A 330, 296 (1993).

2. L. Badano, M. Benedikt, P. Bryant, M. Crescenti, P. Holý, P. Knaus, A. Maier, M. Pullia and S. Rossi, Nucl. Instr. Meth. Phys. Res. A 430, 512 (1999).

3. M. Pavlovič, Nucl. Instr. Meth. Phys. Res. A 438, 548 (1999).

4. M. Pavlovič, E. Griesmayer and R. Seemann, Nucl. Instr. Meth. Phys. Res. A 545, 412 (2005).

5. J. Y. Tang, L. Liu, Z. Yang, S. X. Fang and X. L. Guan, Phys. Rev. STAB 12, 050101 (2009).

6. M. Benedikt, P. Bryant and M. Pullia, Nucl. Instr. Meth. Phys. Res. A 430, 523 (1999).

7. F. T. Cole, P. V. Livdahl, F. E. Mills and L. Teng, Multi-station Proton Beam Therapy System; United States Patent US 4,870,287, 26 September 1989.

8. E. Griesmayer, T. Schreiner and M. Pavlovič, Nucl. Instr. and Meth. B 258, 134 (2007).

9. M. Benedikt, Nucl. Instr. Meth. Phys. Res. A 539, 25 (2005).

10. H. Wiedemann, Particle Accelerator Physics: Basic Principles and Linear Beam Dynamics, $1^{\text {st }}$ edn. (Springer-Verlag, Berlin, Heidelberg, New York, 1993).

11. P. J. Bryant, and K. Johnsen, The Principles of Circular Accelerators and Storage Rings, $1^{\text {st }}$ edn. (Cambridge University Press, Cambridge, GB, 1993). 